\title{
Teste da acurácia de um modelador acústico 2D por diferenças finitas no domínio do tempo
}

\author{
Lucas de C. Costa, Amanda G. Pereira, Emilia C. Hartmann e Daniel L. Macedo, UFPA, Brazil
}

Copyright 2019, SBGf - Sociedade Brasileira de Geofísica.

This paper was prepared for presentation at the $16^{\text {th }}$ International Congress of the Brazilian Geophysical Society, held in Rio de Janeiro, Brazil, August 19-22, 2019.

Contents of this paper were reviewed by the Technical Committee of the $16^{\text {th }}$ International Congress of The Brazilian Geophysical Society and do not necessarily represent any position of the SBGf, its officers or members. Electronic reproduction or storage of any part of this paper for commercial purposes without the written consent of The Brazilian Geophysical Society is prohibited.

\begin{abstract}
In this work we tested the accuracy and the application limit present in one of the modelers of the seismic processing and imaging package, Madagascar. The program used was sfawefd2d: $2 D$ acoustic timedomain FD modeling, which models the acoustic wave in two dimensions as a function of speed and density. These tests were made comparing the numerical and semianalytic solution for different parameters such as: change of relative position of the receivers w.r.t the source and the frequency. Both parameters alowed us to verify the numerical dispersion and anisotropy.
\end{abstract}

\section{Introdução}

A teoria que descreve a propagação de uma onda mecânica é a base da sismologia e sísmica de exploração. Essa teoria está baseada na resolução das equações diferenciais parciais desde o modelo mais simples - onda acústica em um meio com densidade constante - até o modelo com uma complexidade maior. Saber a solução da equação da onda para um dado conjunto ou distribuição de fontes em um dado meio é crucial em várias aplicações dentro da sísmica de exploração: estudos de iluminação para um levantamento sísmico, amarração poço-sísmica, migração RTM, FWI, por exemplo.

Porém, nem sempre esta solução pode ser obtida facilmente. $\mathrm{Na}$ verdade, a não ser em casos mais simples de meios homogêneos ou meios com complexidade muito baixa, a solução analítica nunca está ao alcance de nossas mãos. Neste contexto, a procura por soluções numéricas se torna relevante, o que é comumente conhecido por modelagem numérica. Esta usa modelos matemáticos para descrever as condições físicas de cenários geológicos usando números e equações. Com modelos numéricos, existem técnicas, como métodos de diferenças finitas e elementos finitos, para aproximar as soluções dessas equações. Experimentos numéricos podem então ser realizados nesses modelos, produzindo os resultados que podem ser interpretados no contexto do processo geológico (Ismail-Zadeh and Tackley, 2010).

Neste trabalho, será apresentado os testes em um modelador acústico 2D, com o intuito de comparar o dado modelado com o dado semi-analítico e, desta forma, analisar a acurácia do modelador.
Para que fosse possível a realização desse teste foi necessário aprender sobre o pacote de software opensource, Madagascar, que é utilizado para a análise de dados multidimensionais e experimentos computacionais reproduzíveis (Madagascar, 2019). O aprendizado deste foi por meio de mini curso e por meio de exercícios com diversos programas.

Em seguida, foi necessário o estudo dos pressupostos teóricos. Isto envolveu os fundamentos da equação da onda 2D (Wapenaar, 2014), sua função de Green e solução desta como convolução da mesma com a fonte (Snieder, 1994); as características do modelador, sfawefd2d; o método das diferenças finitas e a discretização da equação da onda (Fichtner, 2010).

Após o estudo do Madagascar e da parte teórica, foram realizados os testes, que consistiram na comparação da resposta modelada com a solução semi-analítica para um meio com velocidade e densidade homogêneas. A solução modelada foi feita a partir de uma fonte pontual com assinatura igual a uma Ricker. A solução semi-analítica foi construída a partir da convolução numérica da fonte usada na modelagem com a função de Green analítica.

Por fim, o modelo foi construído com vários receptores com distâncias e angulações em relação à fonte distintas utilizando um sinal Ricker com diferentes frequências. Estas variações de parâmetros foram idealizadas para analisar a dispersão e anisotropia numérica.

\section{Pressupostos Teóricos}

O Modelador usado é baseado ${ }^{1}$ na equação acústica bidirecional (two-way) 2D com densidade constante

$$
\nabla^{2} P(x, z, t)-\frac{1}{c(x, z)^{2}} \frac{\partial^{2} P(x, z, t)}{\partial t^{2}}=S(x, z, t),
$$

na qual $P(x, z, t)$ é o campo de pressão, $c(x, z)$ é a velocidade de propagação da onda no meio e $S(x, z, t)$ é a fonte.

A função de Green desta equação, para este caso, é (Snieder, 1994)

$$
G^{2 D}(r, t)=\left\{\begin{array}{cc}
0 & \text { para } t<r / c \\
\frac{1}{2 \pi} \frac{1}{\sqrt{t^{2}-r^{2} / c^{2}}} & \text { para } t>r / c
\end{array}\right.
$$

sendo $r=\left\|\boldsymbol{r}-\boldsymbol{r}_{s}\right\|$ onde $\boldsymbol{r}=(x, z)$ e $\boldsymbol{r}_{s}$ é a posição da fonte.

\footnotetext{
${ }^{1} \mathrm{Na}$ verdade, o modelador tem como opção modelar com a discretização da equação (1) ou com a equação

$$
\rho(x, z) \nabla \cdot\left(\frac{1}{\rho(x, z)} \nabla P(x, z, t)\right)-\frac{1}{c(x, z)^{2}} \frac{\partial^{2} P(x, z, t)}{\partial t^{2}}=S(x, z, t),
$$

onde $\rho(x, z)$ é a densidade do meio.
} 
Para encontrar a evolução do campo de onda, deve-se discretizar a equação (1). Esta será feita utilizando-se o método das diferenças finitas, que é caracterizado por substituir as derivadas de uma equação diferencial por expressões algébricas de diferenças. Essas expressões são obtidas a partir do truncamento da expansão em série de Taylor, e a ordem do erro da aproximação da derivada é função desse truncamento. A aplicação do método das diferenças finitas é realizada considerando-se o domínio discretizado em um grid retangular de pontos, geralmente equiespaçados. Essa característica dificulta sua aplicação em domínios curvos. Outra desvantagem é que o método não permite que se tenham grids com refinamentos diferentes sobre o domínio. Esse método de discretização pode ser aplicado na equação da onda acústica escalar (Zakaria et al., 2000). Portanto, sabendo que é uma equação de segunda ordem, pode-se fazer a seguinte aproximação para a derivada parcial em relação à $x$ :

$$
\frac{\partial^{2} P(x, z, t)}{\partial x^{2}} \approx \frac{P_{i+1, j}^{l}-2 P_{i, j}^{l}+P_{i-1, j}^{l}}{\Delta x^{2}},
$$

onde $P_{i, j}^{l}=P\left(x_{i}, z_{j}, t_{l}\right)$ e $x_{i}=x_{0}+i \Delta x, z_{j}=z_{0}+j \Delta z$, e $t_{l}=t_{0}+$ $l \Delta t$. Esta é uma aproximação de segunda ordem. Pode-se, de maneira análoga aproximar as segundas derivadas em $z$ e $t$

Substituindo em (1) as derivadas por suas aproximações de diferenças finitas, e Isolando o termo $P_{i, j}^{l+1}$, temos

$$
\begin{aligned}
P_{i, j}^{l+1}=c^{2} \frac{\Delta t^{2}}{\Delta h^{2}}\left(P_{i+1, j}^{l}+P_{i-1, j}^{l}\right. & \left.+P_{i, j+1}^{l}+P_{i, j-1}^{l}-4 P_{i, j}^{l}\right) \\
& +2 P_{i, j}^{l}-P_{i, j}^{l-1}-c^{2} \Delta t S_{i, j}^{l},
\end{aligned}
$$

onde foi considerado $\Delta x=\Delta z=\Delta h$. Esta equação representa a evolução do campo no tempo.

\section{Relação de Dispersão}

Assumindo a propagação de uma onda plana em um meio homogêneo, temos:

$$
P_{i, j}^{l}=e^{i\left(k_{x} x_{i}+k_{z} z_{j}-\omega t_{l}\right)} .
$$

A partir desta equação é possível determinar as seguintes relações:

$$
\begin{aligned}
P_{i \pm 1, j}^{l} & =e^{i\left[k_{x}\left(x_{i} \pm \Delta x\right)+k_{z} z_{j}-\omega t_{l}\right]} \\
& =e^{ \pm i k_{x} \Delta x} e^{i\left(k_{x} x_{i}+k_{z} z_{j}-\omega t_{l}\right)}=e^{ \pm i k_{x} \Delta x} P_{i, j}^{l}
\end{aligned}
$$

De modo análogo

$$
P_{i, j \pm 1}^{l}=e^{ \pm i k_{z} \Delta x} P_{i, j}^{l}
$$

e

$$
P_{i, j}^{l \pm 1}=e^{\mp i \omega \Delta t} P_{i, j}^{l} .
$$

Substituindo as equações (6), (7) e (8) em (4), considerando $S_{i, j}^{l}=0$, tem-se que:

$$
\begin{aligned}
e^{-i \omega \Delta t} P_{i, j}^{l}+e^{i \omega \Delta t} P_{i, j}^{l}= & c^{2} \frac{\Delta t^{2}}{\Delta x^{2}}\left(e^{+i k_{x} \Delta h} P_{i, j}^{l}\right. \\
& +e^{-i k_{x} \Delta h} P_{i, j}^{l}+e^{+i k_{z} \Delta h} P_{i, j}^{l} \\
& \left.+e^{-i k_{z} \Delta h} P_{i, j}^{l}-4 P_{i, j}^{l}\right)+2 P_{i, j}^{l}
\end{aligned}
$$

A partir da relação de Euler para cosseno,

$$
\cos (y)=\frac{e^{i y}+e^{-i y}}{2},
$$

podemos chegar na seguinte equação

$$
\cos (\omega \Delta t)=c^{2} \frac{\Delta t^{2}}{\Delta h^{2}}\left(\cos \left(k_{x} \Delta h\right)+\cos \left(k_{z} \Delta h\right)-2\right)+1
$$

Sendo $k=\frac{\omega}{c}$ o número de onda, podemos definir $k_{x}=$ $k \sin \theta$ e $k_{z}=k \cos \theta$, onde $-\pi<\theta<\pi$ é a direção de propagação da onda plana em relação ao eixo vertical, orientado para baixo. Com isso, Equação (11) fica

$$
\cos (\omega \Delta t)=c^{2} \frac{\Delta t^{2}}{\Delta h^{2}}(\cos (k \sin \theta \Delta h)+\cos (k \cos \theta \Delta h)-2)+1 .
$$

Da eq. (12) podemos escrever

$$
\begin{aligned}
\omega(k, \theta)= & \frac{1}{\Delta t} \cos ^{-1}\left\{c^{2} \frac{\Delta t^{2}}{\Delta h^{2}}(\cos (k \sin \theta \Delta h)\right. \\
& +\cos (k \cos \theta \Delta h)-2)+1\} .
\end{aligned}
$$

Desta equação podemos escrever as expressões para velocidade de fase $\left(c_{p}\right)$ e de grupo $\left(c_{g}\right)$ :

$$
\begin{gathered}
c_{p}(f, \theta)=\frac{c}{2 \pi f \Delta t} \cos ^{-1}\left\{c ^ { 2 } \frac { \Delta t ^ { 2 } } { \Delta h ^ { 2 } } \left(\cos \left(\frac{2 \pi f}{c} \sin \theta \Delta h\right)\right.\right. \\
\left.\left.+\cos \left(\frac{2 \pi f}{c} \cos \theta \Delta h\right)-2\right)+1\right\}
\end{gathered}
$$

e

$$
\begin{aligned}
c_{g}(f, \theta)= & \left(\frac{c^{2} \Delta t}{\Delta h}[\sin (k \sin \theta \Delta h) \sin \theta\right. \\
& +\sin (k \cos \theta \Delta h) \cos \theta]) \times \\
& \left(1-\left\{c^{2} \frac{\Delta t^{2}}{\Delta h^{2}}(\cos (k \sin \theta \Delta h)\right.\right. \\
& \left.+\cos (k \cos \theta \Delta h)-2)+1\}^{2}\right)^{-1 / 2}
\end{aligned}
$$

Os gráficos de dispersão para essas equações são apresentados na Figura 1.

\section{Metodologia}

No primeiro experimento foi utilizado um modelo com $1000 \mathrm{~m}$ de largura e $1000 \mathrm{~m}$ de profundidade, sendo constituído por um meio com densidade e velocidade $(1500 \mathrm{~m} / \mathrm{s})$ constantes e frequência de $10 \mathrm{~Hz}$. Na modelagem usamos o espaçamento de $2 \mathrm{~m}$ tanto no eixo $x$ quanto no eixo $z$ e o passo no tempo de $0.0004 s$. Na sua geometria de aquisição, foram usados 15 receptores espaçados de $100 \mathrm{~m}, 450 \mathrm{~m}$ e $800 \mathrm{~m}$ da fonte, com angulações de $0^{\circ}, 15^{\circ}, 30^{\circ}, 45^{\circ}$ e $90^{\circ}$ em relação ao eixo $z$ (Fig. 2). A numeração dos receptores pode ser vista na Tabela 1. O objetivo de deixar os receptores em diferentes distâncias e angulações é para analisar a dispersão e a anisotropia numérica, respectivamente. 


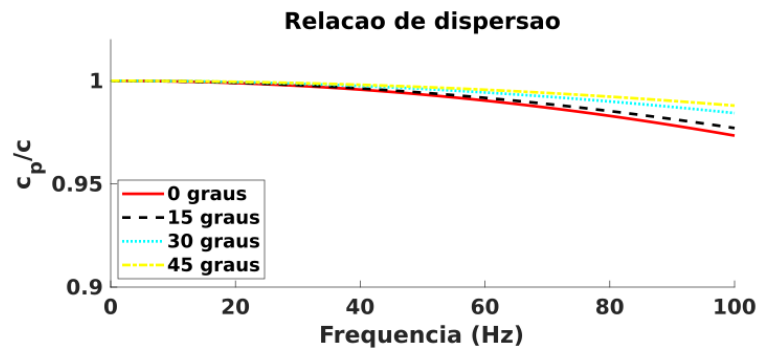

(a) Velocidade de fase sobre velocidade do meio em X Frequência

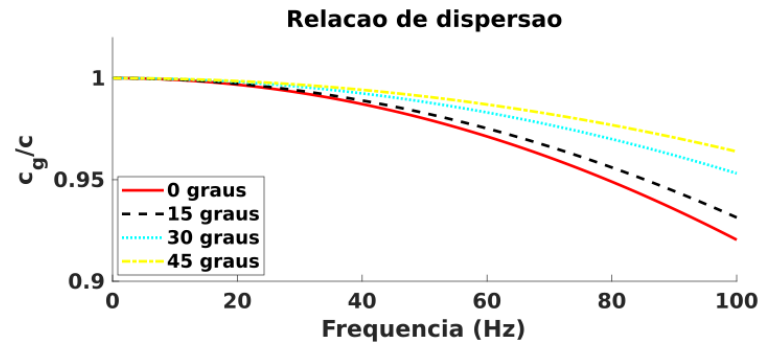

(b) Velocidade de grupo sobre velocidade do meio X Frequência

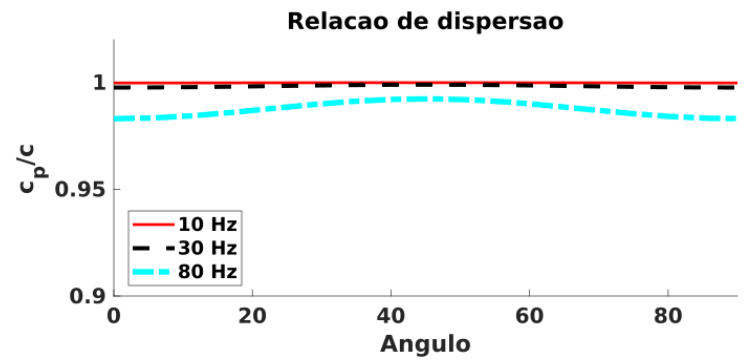

(c) Velocidade de fase sobre velocidade do meio em X Ângulo

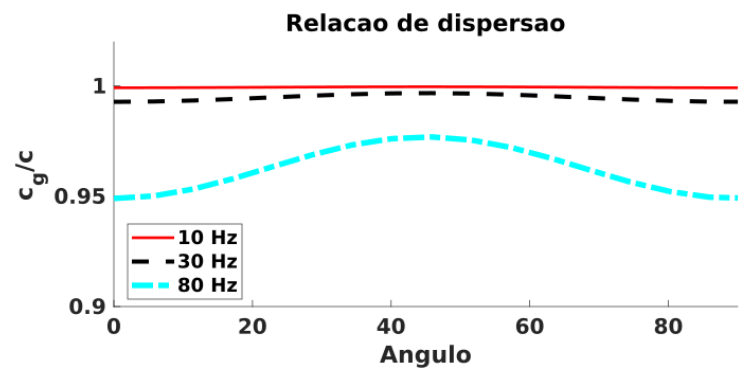

(d) Velocidade de grupo sobre velocidade do meio X Ângulo

Figure 1: Diagramas de dispersão, considerando a frequência como variável para a) e b) e o ângulo para c) e d).

O segundo experimento consistiu em alterar as dimensões em relação ao primeiro. Isto ocorreu devido a interferência de reflexões indesejadas na borda do modelo anterior. $O$ novo modelo deixou os receptores nas mesmas posições, porém suas dimensões foram alteradas: de $-1000 \mathrm{~m}$ a $2000 \mathrm{~m}$ tanto na vertical como na horizontal. O objetivo é deixar a fonte e o arranjo de receptores centralizados, para que reflexões indesejadas nas bordas não influenciem o sinal nos receptores.

Para os outros experimentos, foi utilizado o segundo modelo como base. O parâmetro variado foi a frequência. Foram utilizadas três frequências diferentes $(10 \mathrm{~Hz}, 30 \mathrm{~Hz}$

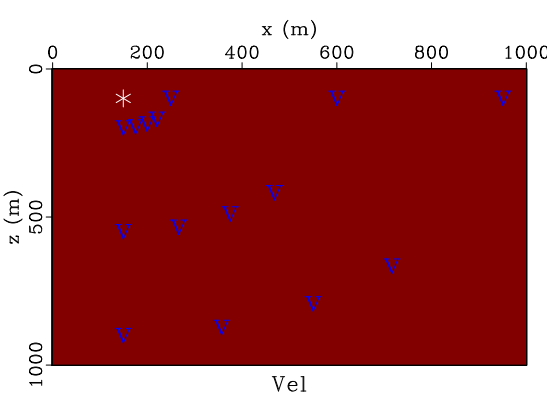

Figure 2: Primeiro modelo com velocidade e densidade constante.

e $80 \mathrm{~Hz}$ ), com o intuito de analisar como ocorre a dispersão com cada uma destas frequências.

Uma maneira de avaliar a qualidade é olhar traço a traço, da resposta modelada e do semi-analítico. No entanto, para sintetizar a informação de maneira mais eficiente, foram gerados dois tipos de painéis de comparação:

\section{Amplitude máxima relativa $\times$ tempo de trânsito}

para cada traço, modelado e semi-analítico, foi identificado o ponto de maior amplitude em módulo e o correspondente tempo de trânsito. As amplitudes foram normalizadas pelo valor obtido no traço semianalítico correspondente. Para cada experimento, cinco paineis são gerados, cada um comparando receptores correspondentes a um mesmo ângulo;

Energia total relativa $\times$ número do traço a energia total é calculada a partir do quadrado do módulo do dado que, em seguida, é empilhado em uma dimensão. As energias também foram normalizadas pela energia do traço semi-analítico correspondente. Para cada experimento 1 painel é gerado com as energias de todos os traços modelados e semi-analíticos. A numeração segue a Tabela 1 .

Este paineis são importantes pois irão facilitar a análise dos dados, sendo mais simples de visualizar fenômenos como anisotropia e dispersão numérica.

Table 1: A numeração de cada receptor, sinalizando a distância e o ângulo em relação à fonte.

\begin{tabular}{|c|c|c|c|c|c|}
\hline & $0^{\circ}$ & $15^{\circ}$ & $30^{\circ}$ & $45^{\circ}$ & $90^{\circ}$ \\
\hline $100 \mathrm{~m}$ & 1 & 4 & 7 & 10 & 13 \\
\hline $450 \mathrm{~m}$ & 2 & 5 & 8 & 11 & 14 \\
\hline $800 \mathrm{~m}$ & 3 & 6 & 9 & 12 & 15 \\
\hline
\end{tabular}

\section{Resultados}

$\mathrm{Na}$ Figura 3(b), mostra-se a resposta modelada (vermelho) sobre a resposta semi-analítica (azul). Esta última foi construída pela convolução discreta da fonte (Ricker de $10 \mathrm{~Hz}$ ) com a função de Green correspondente (Figura 3(a)). O valor final da convolução foi ponderado pelo $\Delta t, \Delta x$ e $\Delta z$. Este resultado mostra um "casamento" quase perfeito entre o dado modelado e o semi-analítico.

$\mathrm{Na}$ Figura 4 podemos ver os traços nos receptores à $800 \mathrm{~m}$ e ângulos de $0^{\circ}$ e $90^{\circ}$. Veja que a concordância 


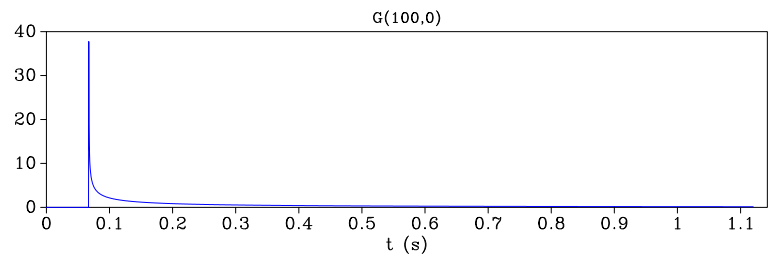

(a) Função de Green

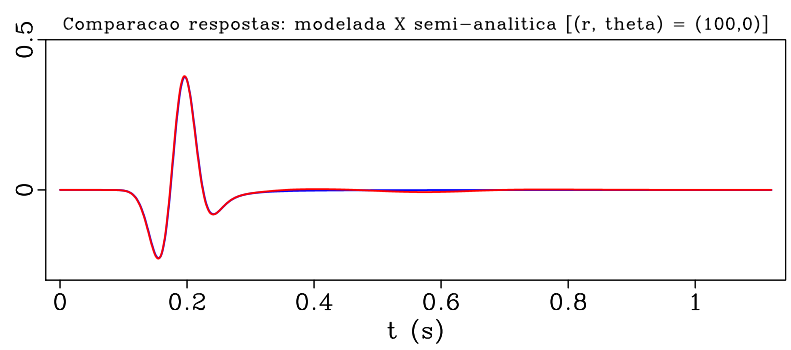

(b) Comparação entre resposta modelada e semi-analítica

Figure 3: Receptor à $100 \mathrm{~m}$ da fonte no angulo de $0^{\circ}$ no modelo sem bordas extendidas. Em vermelho, a resposta modelada, e em azul a resposta semi-analítica. Esta convenção será usada ao longo de todo o resumo.

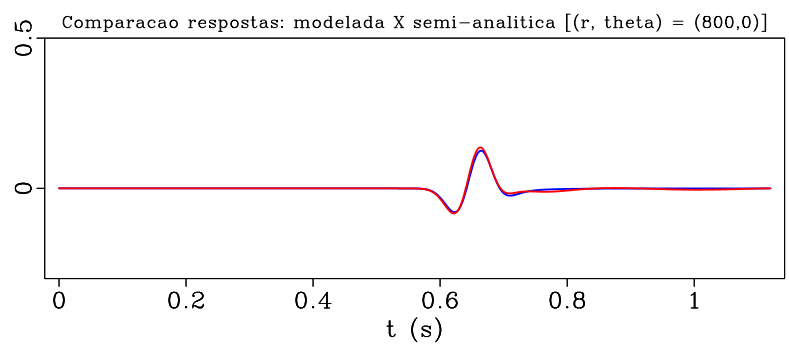

(a) Receptor $(r, \theta)=\left(800,0^{\circ}\right)$

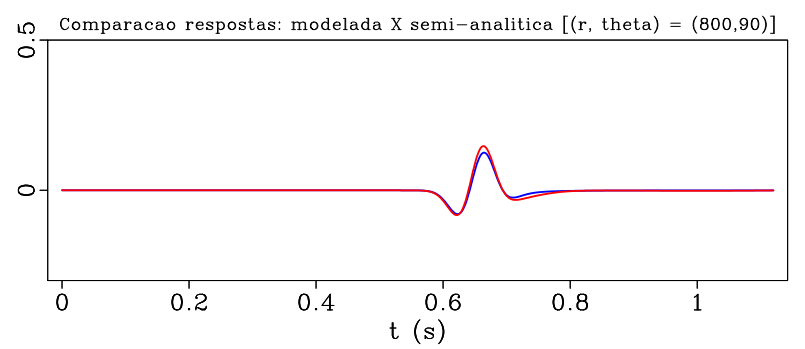

(b) Receptor $(r, \boldsymbol{\theta})=\left(800,90^{\circ}\right)$

Figure 4: Receptores à $800 \mathrm{~m}$ da fonte nos ângulos de (a) $0^{\circ}$ e (b) $90^{\circ}$ no modelo sem bordas extendidas. Note a influência das reflexões indesejadas nas bordas.

dos traços modelados e semi-analíticos não tem a mesma qualidade que a observada na Figura 3(b). Nossa hipótese é que a borda absorvente implementada, baseada na equação unidirecional (one-way), não absorve perfeitamente os campos incidentes dando origem a reflexões não desejadas.

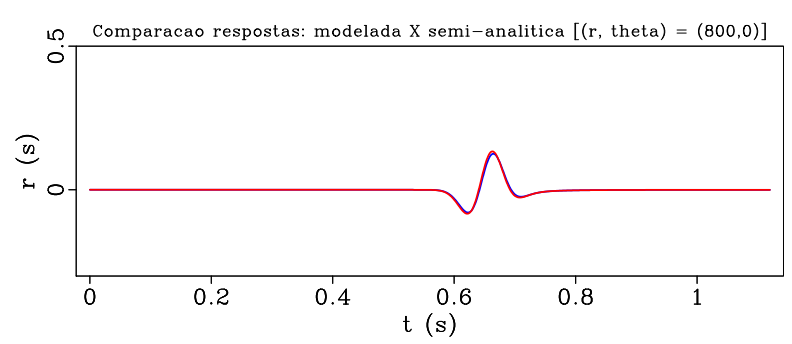

(a) Receptor $(r, \theta)=\left(800,0^{\circ}\right)$.

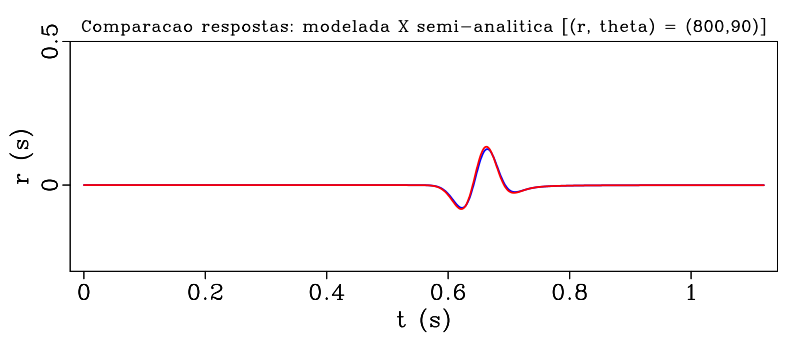

(b) Receptor $(r, \boldsymbol{\theta})=\left(800,90^{\circ}\right)$.

Figure 5: Receptores à $800 \mathrm{~m}$ da fonte nos ângulos de (a) $0^{\circ}$ e (b) $90^{\circ}$ no modelo com bordas extendidas. Note que a concordância neste caso é melhor que a observada na Figura 4.

Para testar a hipótese acima foi analisada como se comporta a resposta para o mesmo arranjo e para os mesmos receptores, mas utilizando bordas extendidas (Fig. 5). Em comparação com a Figura 4, nota-se que os valores modelados estão mais parecidos com os semianalíticos, sem a presença de muitas oscilações. Este resultado significa que o modelador não possui bordas absorventes perfeitas, o que ocasiona a interferência de reflexões na modelagem dos dados para modelos menores, como o mostrado na Figura 2.

Para fazer uma análise geral de cada receptor, foram gerados gráficos comparativos. Estes variam com a angulação, distância do receptor para fonte e frequência.

$\mathrm{Na}$ Figura 6, nota-se que a comparação do tempo de trânsito com a amplitude máxima não varia com angulação para esta frequência $(10 \mathrm{~Hz})$. O mesmo pode ser visto na Figura 7: a uma mesma distância da fonte, mas com diferentes ângulos, observamos a mesma energia. Isto indica que para esta frequência, não há anisotropia numérica com era esperado pelo diagrama de dispersão mostrado na Figura 1.

O dado modelado com maior diferença do semi-analítico é o encontrado à $450 \mathrm{~m}$ da fonte(Fig. 8). Isto contraria o que se esperava, já que essa diferença deveria aumentar com a distância (Figura 1). Bom salientar que isto acontece para todas as frequências seguintes.

Na Figura 9, observa-se que os resultados de comparação do tempo de trânsito com a amplitude máxima variam com a angulação quando o meio possui uma frequência de $30 \mathrm{~Hz}$. Este fato é mais facilmente observado para os receptores à $100 \mathrm{~m}$. A análise dos receptores que possuem inclinação de $0^{\circ}$ e $90^{\circ}$ são similares e diferentes das 

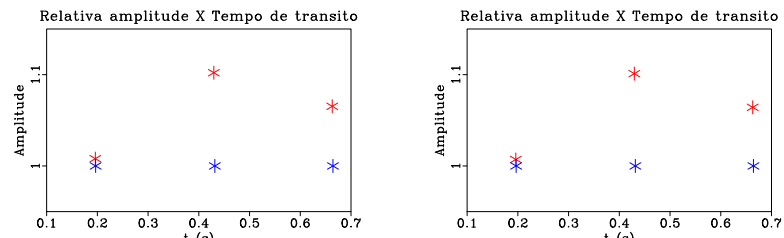

(a) $(\theta)=\left(0^{\circ}\right)$.

(b) $(\theta)=\left(15^{\circ}\right)$
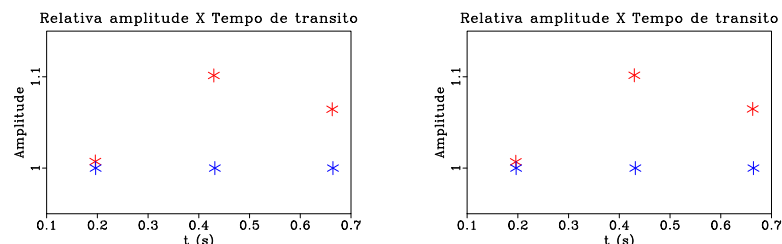

(c) $(\theta)=\left(30^{\circ}\right)$.

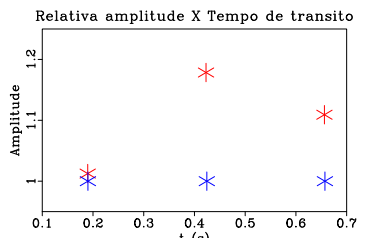

(a) $(\theta)=\left(0^{\circ}\right)$

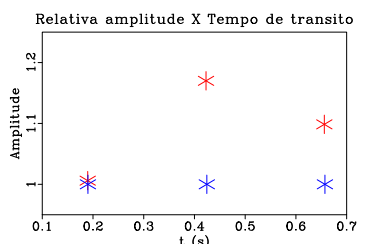

(c) $(\theta)=\left(30^{\circ}\right)$

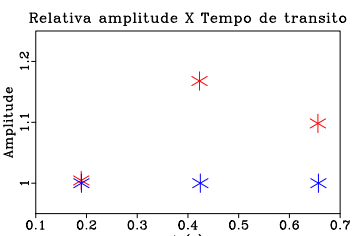

(b) $(\theta)=\left(15^{\circ}\right)$.

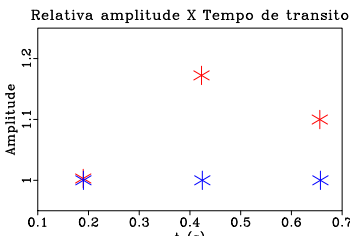

(d) $(\theta)=\left(45^{\circ}\right)$.

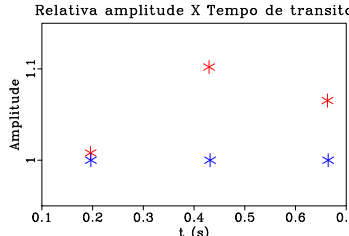

(e) $(\theta)=\left(90^{\circ}\right)$.

Figure 6: Comparação do tempo de trânsito com a amplitude para o modelo com frequência de $10 \mathrm{~Hz}$.

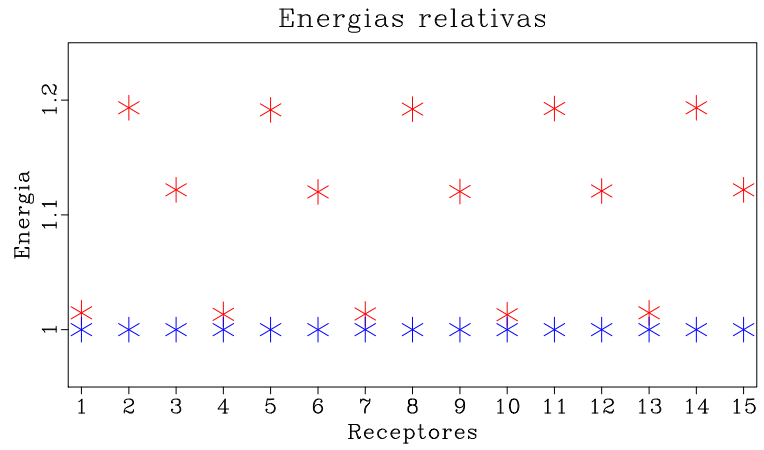

Figure 7: Comparação relativa de energia para $10 \mathrm{~Hz}$.

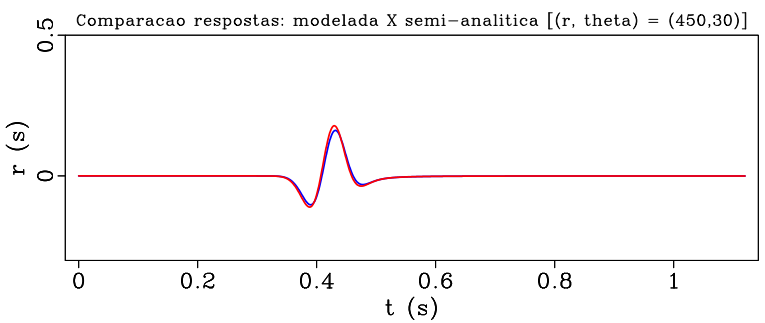

Figure 8: Receptor à $450 \mathrm{~m}$ da fonte no ângulo de $45^{\circ}$. Note que o traço modelado possui diferença notória do dado semi-analítico, diferente do que ocorre na Figura 5.

demais inclinações. Isto indica que para esta frequência

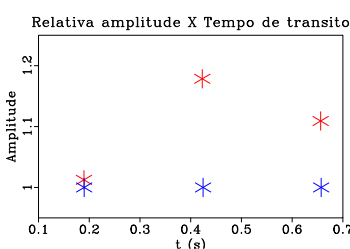

(e) $(\theta)=\left(90^{\circ}\right)$.

Figure 9: Comparação do tempo de trânsito com a amplitude para o modelo com frequência de $30 \mathrm{~Hz}$.

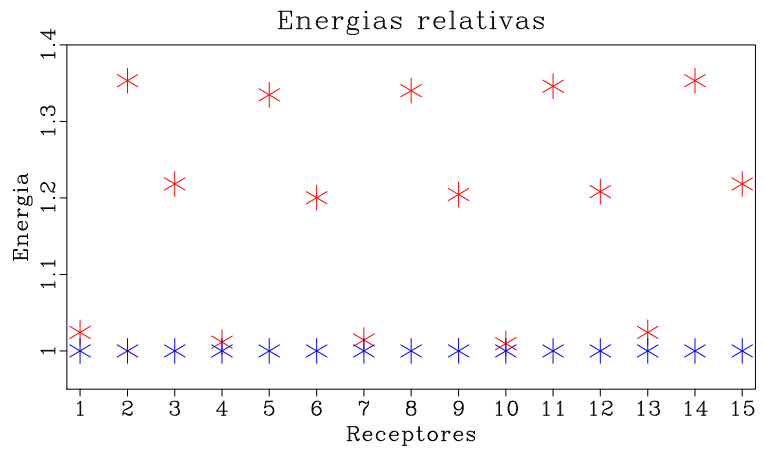

Figure 10: Comparação relativa de energia para $30 \mathrm{~Hz}$.

há anisotropia numérica como era esperado pelo diagrama de dispersão mostrado na Figura 1.

A figura 10 reforça a análise acima, pois para receptores com mesma distância da fonte, mas com diferentes inclinações existe uma mudança sutil de energia.

Já a Figura 11, que está relacionada com os resultados da frequência $80 \mathrm{hz}$, percebe-se que a comparação do tempo de trânsito com a amplitude máxima variam com a angulação. Assim como na Figura 9, as inclinaçoes de $0^{\circ} \mathrm{e}$ $90^{\circ}$ são similares e diferentes das demais inclinações. Isto indica que para esta frequência há anisotropia numérica como era esperado pelo diagrama de dispersão mostrado na Figura 1.

A Figura 12 mostra que para receptores com mesma distância da fonte, mas com diferentes inclinações 


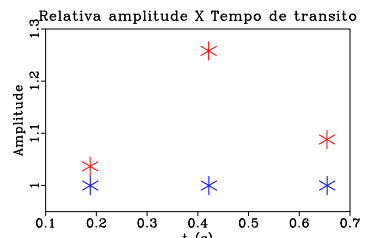

(a) $(\theta)=\left(0^{\circ}\right)$.

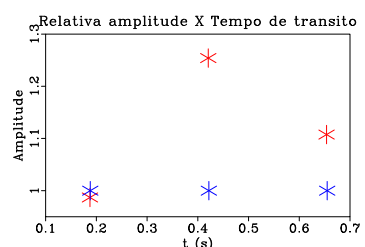

(c) $(\theta)=\left(30^{\circ}\right)$.

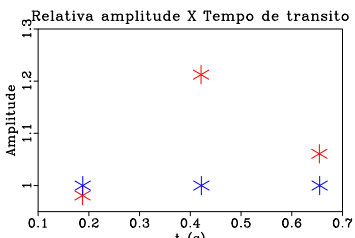

(b) $(\theta)=\left(15^{\circ}\right)$.

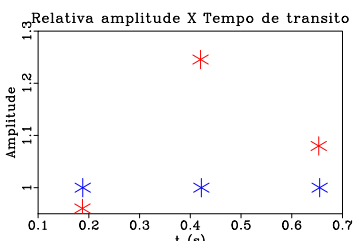

(d) $(\theta)=\left(45^{\circ}\right)$.

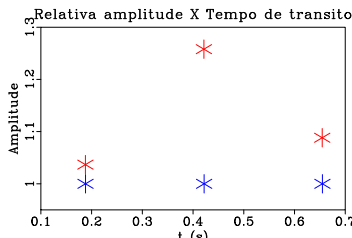

(e) $(\theta)=\left(90^{\circ}\right)$.

Figure 11: Comparação do tempo de trânsito com a amplitude para o modelo com frequência de $80 \mathrm{~Hz}$.

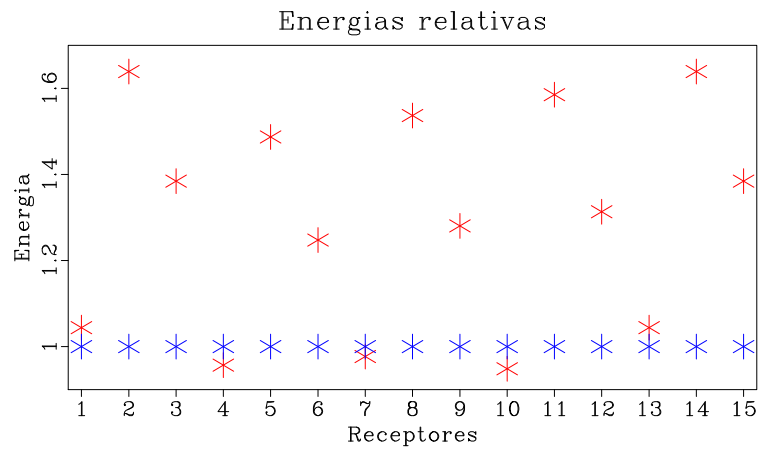

Figure 12: Comparação relativa de energia para $80 \mathrm{~Hz}$.

ocorre uma mudança bruta de energia, deixando a "linha" dos receptores mais curvada. Isso significa que esta frequência apresenta uma alta dispersão, como representada na Figura 13.

\section{Conclusão}

A partir dos resultados deste trabalho, conclui-se que o modelador sfawefd2d possui um bom funcionamento. A maioria dos resultados de nossos experimentos numéricos teve boa correlação com o esperado. Além disso, os experimentos corroboraram as relações de dispersão teóricas.

Podemos avaliar que a diferença dos resultados é baixa para os receptores com a mesma distância da fonte e com ângulos diferentes. Quanto maior a frequência,

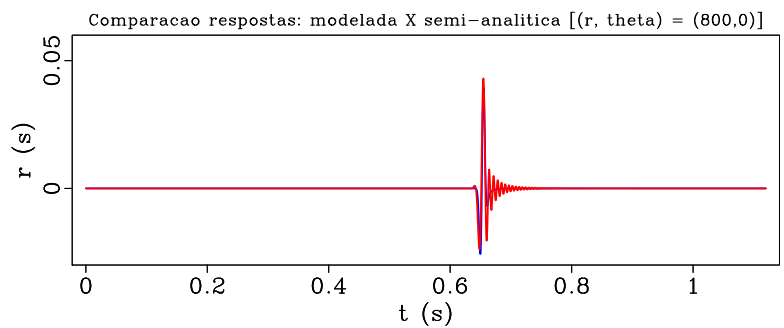

(a) Receptor $(r, \theta)=\left(800,0^{\circ}\right)$.

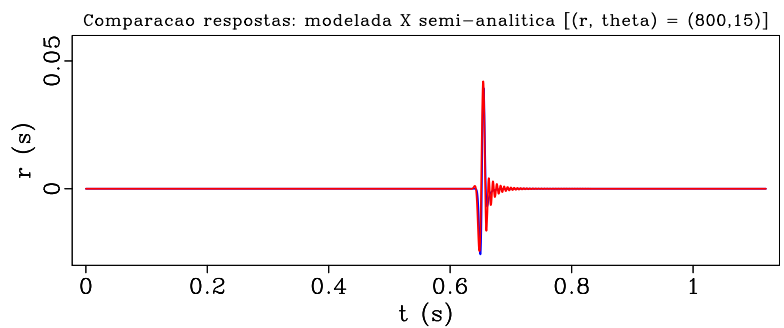

(b) Receptor $(r, \theta)=\left(800,15^{\circ}\right)$.

Figure 13: Receptores à $800 \mathrm{~m}$ da fonte nos ângulos de (a) $0^{\circ}$ e (b) $15^{\circ}$ para o experimento com a frequência de $30 \mathrm{~Hz}$. Note a dispersão numérica que ocorre nos dados para esta faixa de frequência.

a diferença torna-se um pouco maior. Sendo assim, podemos afirmar que o modelo de $10 \mathrm{~Hz}$ possui isotropia numérica, enquanto os outros modelos possuem uma baixa anisotropia numérica.

Os dados que deram diferentes do esperado foram para a distância de $450 \mathrm{~m}$ em relação a fonte. Esperávasse que a maior diferença do dado semi-analítico ocorresse para o dado de $800 \mathrm{~m}$ da fonte, o que não ocorreu na prática.

\section{References}

Fichtner, A., 2010, Full seismic waveform modelling and inversion: Springer Science \& Business Media.

Ismail-Zadeh, A., and Tackley, P., 2010, Computational methods for geodynamics: Cambridge University Press.

Madagascar, 2019. Available on: http://www.ahay.org/wiki/Main_Page. Acess on: March 13th, 2019.

Snieder, R., 1994, A Guided Tour of Mathematical Physics: Samizdat Press, Golden.

Wapenaar, C. P. A., 2014, Elastic wave field extrapolation: Redatuming of single-and multi-component seismic data:, volume 2 Elsevier.

Zakaria, A., Penrose, J., Thomas, F., and Wang, X., 2000, The two dimensional numerical modeling of acoustic wave propagation in shallow water: The two dimensional numerical modeling of acoustic wave propagation in shallow water:, Australian Acoustical Society Conference (Acoustics). 